\title{
No radiographic wrist damage after treatment to target in recent-onset juvenile idiopathic arthritis
}

\author{
P. C. E. Hissink Muller ${ }^{1,2^{*}+}$ D, W. G. van Braak ${ }^{3+}$, D. Schreurs ${ }^{1 \dagger}$, C. M. Nusman ${ }^{3,4}$, S. A. Bergstra ${ }^{8}$, R. Hemke ${ }^{3}$, \\ D. Schonenberg-Meinema ${ }^{4}$, J. M. van den Berg ${ }^{4}$, T. W. Kuijpers ${ }^{4}$, Y. Koopman-Keemink ${ }^{5}$, M. A. J. van Rossum ${ }^{6,7}$, \\ L. W. A. van Suijlekom-Smit ${ }^{2}$, D. M. C. Brinkman' ${ }^{1}$ C. F. Allaart ${ }^{8}$, R. ten Cate $^{1+}$ and M. Maas ${ }^{3+}$
}

\begin{abstract}
Background: To evaluate radiographic progression of patients with new-onset juvenile idiopathic arthritis (JIA) in response to an early, tightly-controlled, treatment-to-target.

Methods: Patients with JIA participating in the BeSt-for-Kids-study, randomized to 3 treatment strategy arms, were eligible if at least 1 conventional wrist-radiograph was available. Bone damage as reflected by carpal length was assessed using the Poznanski-score. The BoneXpert-method was used to determine the Bone Age (BA, > 5 years) and bone mineral density (BMD) of the wrist. These scores were evaluated over time and compared between the treatment arms and mean JADAS10-score using linear mixed models corrected for age and symptom duration.
\end{abstract}

Results: In 60 patients, 252 radiographs were analysed. Baseline age and symptom duration were different between the arms. No difference in comparison to the healthy reference population was found at baseline for the Poznanskiscore (IQR varying from $-0,82 ; 0.68$ ), nor for BA (varying from -0.88 to 0.74 ). Baseline BMD was statistically significantly lower in arm 3 (initial treatment with etanercept and methotrexate) $(-1.48 ;-0.68)$ compared to arm $1(-0.84 ;-0.04)$ and arm $2(-0.93 ; 0.15)$. After treatment to target inactive disease, the Poznanski-scores and the BA remained clinically unchanged, while the BMD in arm 3 improved ( $p<0.05$ vs arm 1).

Conclusions: Recent-onset JIA patients, treated-to-target aimed at inactive disease, showed no signs of radiographic wrist damage (Poznanski-score, BA or BMD) either at baseline or at follow-up, irrespective of treatment arm. A lower BMD at baseline in arm 3, initially treated with methotrexate and etanercept, improved significantly after treatment.

Trial registration: NTR, NL1504 (NTR1574). Registered 01-06-2009.

Keywords: Juvenile idiopathic arthritis, Treatment to target, Radiographic outcome, Conventional radiography

\section{Background}

Juvenile idiopathic arthritis (JIA) is a potentially chronic disease that comprises 7 categories of childhood arthritis of unknown cause, that persists for more than 6 weeks and starts before the age of 16 [1]. Osteopenia, bony

\footnotetext{
* Correspondence: p.hissinkmuller@lumc.nl

${ }^{+}$PCE Hissink Muller, WG van Braak and D Schreurs shared first author.

${ }^{\dagger} \mathrm{R}$ ten Cate and M Maas shared last author.

${ }^{1}$ Department of Pediatric Rheumatology, Leiden University Medical Center, Leiden, the Netherlands

${ }^{2}$ Department of Pediatrics/Pediatric Rheumatology, Sophia Children's

Hospital Erasmus Medical Center, J6-S, LUMC, PO Box 9600, 2300 RC Leiden,

Rotterdam, the Netherlands

Full list of author information is available at the end of the article
}

deformity, erosions, and cartilage loss in carpalia, resulting in carpus shortening, can be complications of inflammation in JIA patients [2-5]. Previous studies have shown that early damage on conventional radiography is correlated with functional deterioration and radiographic progression after 5 years $[2,5,6]$, and also with smaller chances to achieve clinical remission [7]. Monitoring of radiographic damage progression is therefore important to evaluate treatment effect and predict prognosis. Since joint damage is assumed to be the result of ongoing inflammation, reaching inactive disease as early as possible and thereby preventing structural joint damage and consequently limitations in physical functioning, should be 
the goal of treatment [8]. This is facilitated by the availability of new effective disease modifying antirheumatic drugs (DMARDs) [9]. In accordance, current JIA treatment recommendations focus on earlier introduction of DMARDs aiming to achieve remission or at least low disease activity $[8,9]$.

We have recently performed a randomized clinical trial using the treatment-to-target approach in recent-onset JIA patients, comparing 3 strategy-arms with different initial and subsequent treatment steps, aiming at inactive disease, including tapering and stopping DMARD therapy $[10,11]$. In this population we studied radiographic wrist damage using the Poznanski-score, at baseline and evaluated whether damage occurred or recovered with the abrogation of inflammation in the 3 strategy-arms. In addition we used the BoneXpert-method to determine the Bone Age (BA) and Bone Mineral Density (BMD) as markers for joint damage [12].

\section{Methods}

\section{Patient selection}

The Best-for-Kids-study (NTR 1574), a multicenter randomized single-blinded clinical trial, was designed to investigate the effectiveness of three different treatmentstrategies in newly diagnosed patients with the following JIA categories: oligoarticular JIA, rheumatoid factor (RF) negative polyarticular JIA and juvenile psoriatic arthritis. DMARD-naive patients with a disease duration of less than 18 months were randomized to one of the three treatment arms.

Patients in arm 1 were treated with initial monotherapy with methotrexate (MTX) or sulfasalazine (SSZ); patients in arm 2 were treated with initial MTX and prednisone bridging and patients in arm 3 were initially treated with etanercept and MTX. Patients were treated to target, aimed at inactive disease, with three-monthly assessments. If predefined targets of suppression of inflammation were not met, treatment was intensified, as can be seen in Fig. 1, with subsequent treatment-steps, including etanercept also in arm 1 and 2. In case of at least 6 months of inactive disease, treatment was tapered. The current sub-analysis was done in all patients who had radiographs of one or both hands obtained at study inclusion (with a range of maximum 4 months before) or at any follow-up visit up to 40 months. Radiographs of hands and wrists were encouraged at baseline, year 1 and year 2. In practice, physicians were reluctant to do this if there was no local arthritis. Juvenile Arthritis Disease Activity Score (JADAS)10-scores were available from all the patients [11]. To investigate the effect of the relatively fast changing disease activity on slower changing radiological outcome parameters, we have used mean JADAS10scores over 2 years' time as a predictor for the radiological outcomes.
The BeSt-for-Kids-study was approved by the Institutional Review Board at Leiden University Medical Center and written informed consent was obtained from all participants before enrollment.

\section{Radiographic scoring}

All radiographs were anonymized and randomized by an independent computer-technician, and then evaluated using two different scoring methods: the Poznanskiscore [13] and the BoneXpert-method. When radiographs of both wrists were available, scores of both wrists were included. The Poznanski-score was used to measure carpal size, and was calculated as the mean score of 2 independent readers (DS and WB), who were unaware of clinical data. Open growth plates are necessary to determine the Poznanski-score. The radiometacarpal length (RM, defined as the line from the midgrowth plate of the radius to the center of the proximal end of the third metacarpal) and the length of the second metacarpal (M2, defined as the maximum length of the second metacarpal as defined by Garn [14]) were measured, in millimeters using RadiAnt DICOM viewer version 2.2.8, as shown in Fig. 2. Poznanski's gender-specific formulas were used to calculate the expected RM for the observed M2 [13]. The difference between expected and measured RM was then calculated and converted into a Z-score [13], which represents the number of standard deviations that the observed RM diverges from the expected RM. A negative Z-score indicates delayed growth in the radiometacarpal bones with loss of cartilage or loss of joint space as potential causes, whereas a positive Poznanski-score may indicate growth acceleration, a phenomenon thought to be caused by early ossification of carpal bones under influence of chronic hyperemia and inflammation [15]. Radiographic bone damage progression was determined by calculating the change in Z-score between the baseline and followup radiographs.

Next, all radiographs were imported as DICOM-files in the BoneXpert-software for automatic assessment of the BA (using the average Greulich and Pyle bone age [16]), and BMD (BoneXpert Version 2.1.0.12; Visiana, Holte, Denmark). The software generates Z-scores of the BA computed relative to provided scores of healthy children of equal gender, age ( $>5$ years) and ethnicity. A negative Z-score for BA reflects a delayed bone maturation $[12,17]$ whereas a positive Z-score reflects enhanced focal maturation, also possible due to inflammation [15]. The BMD is automatically determined by measuring the amount of cortical bone in the shafts of metacarpal 2-4. The Z-score of BMD is computed compared to provided scores in healthy children of the same bone age and gender. A negative Z-score indicates a diminished BMD. 


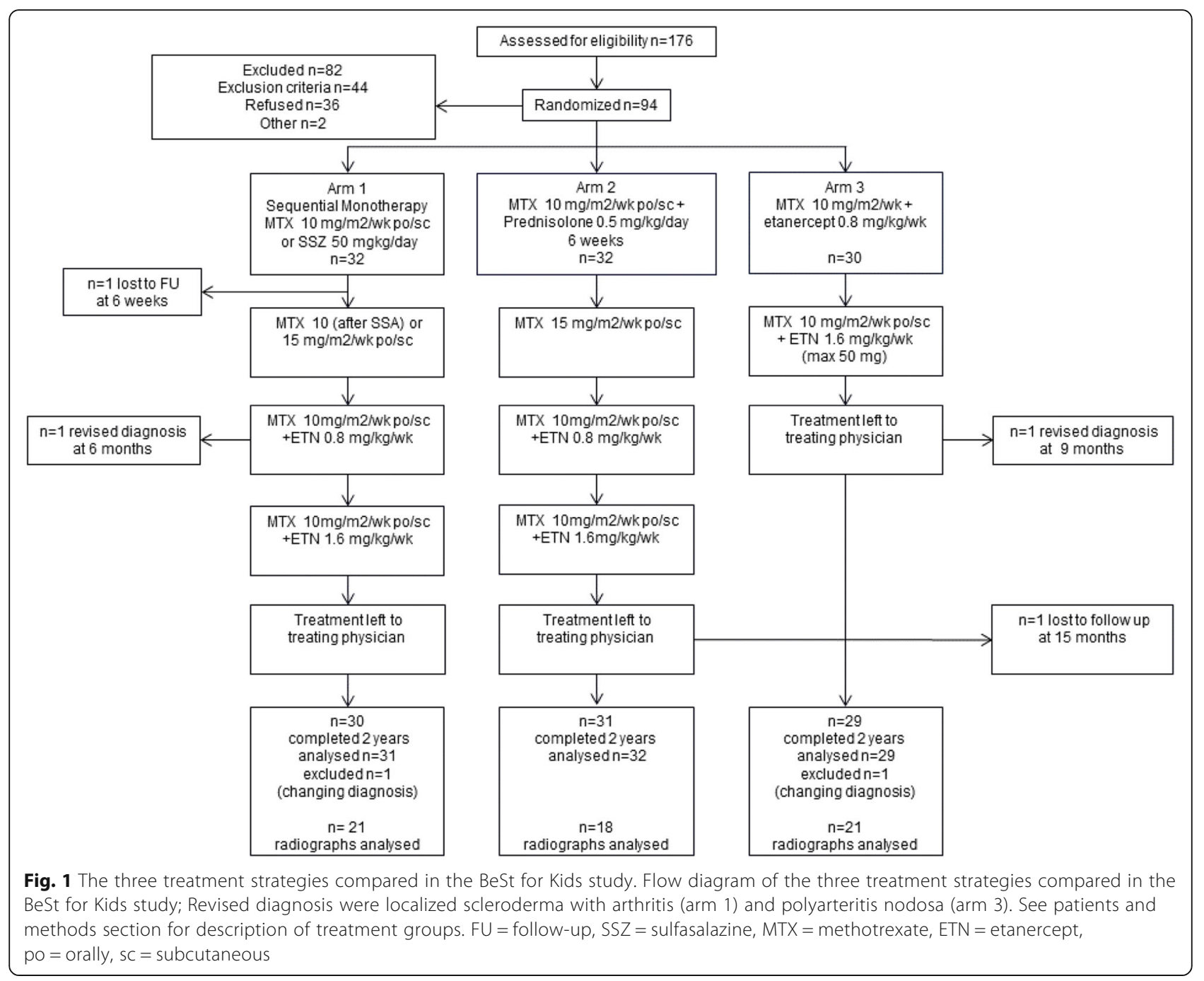

For the Z-scores, the normal population has a normal (Gaussian) distribution around 0.

\section{Statistical analyses}

The single measure intra-class correlation coefficient (ICC) and Bland-Altman plots with 95\% Confidence Interval (CI) were used to determine the agreement of measurements of Poznanski between the two observers and to determine the inter-reader reliability. Baseline characteristics were compared using one-way analysis of variance, Kruskal-Wallis tests or Pearson Chi-square tests, as appropriate. Linear mixed model (LMM) analyses were performed to evaluate the Z-scores of the Poznanskiscore, Bone Age and BMD over time between the 3 treatment groups. LMM was also used to evaluate the different Z-scores over time for the mean JADAS-10 score over 2 years' time, since we assumed that average disease activity over 2 years' time could have an effect on slower changing variables like Poznanski score, Bone Age and BMD. We assumed a multilevel structure of measurements over time (level 1), nested within hands (left or right, level 2), nested within patients (level 3), and added a random intercept and slope to take into account correlations of measurements performed within the same hand within the same patient and differences in time periods between the different radiographs. For the Poznanski-score, the model was adjusted for the potential baseline confounders age and duration of symptoms. Since the BA and BMD account for age in itself, the models for BA and BMD were adjusted for duration of symptoms only. Multiple imputation using package mice in software package $\mathrm{R}$ was used to deal with missing values for symptom duration and JADAS10-score, with $n=10$ imputed data sets [11].

For all statistical analyses a $p$-value $<0.05$ was considered statistically significant. A deviation of $>1$ in Z-score, indicating a deviation $>1 S D$ from the mean in a normal population, was arbitrarily defined as clinically relevant [17]. Statistical analyses were performed with SPSS version 23 software (SPSS, Chicago, IL., USA) and Stata SE version 14. (StataCorp LP). 


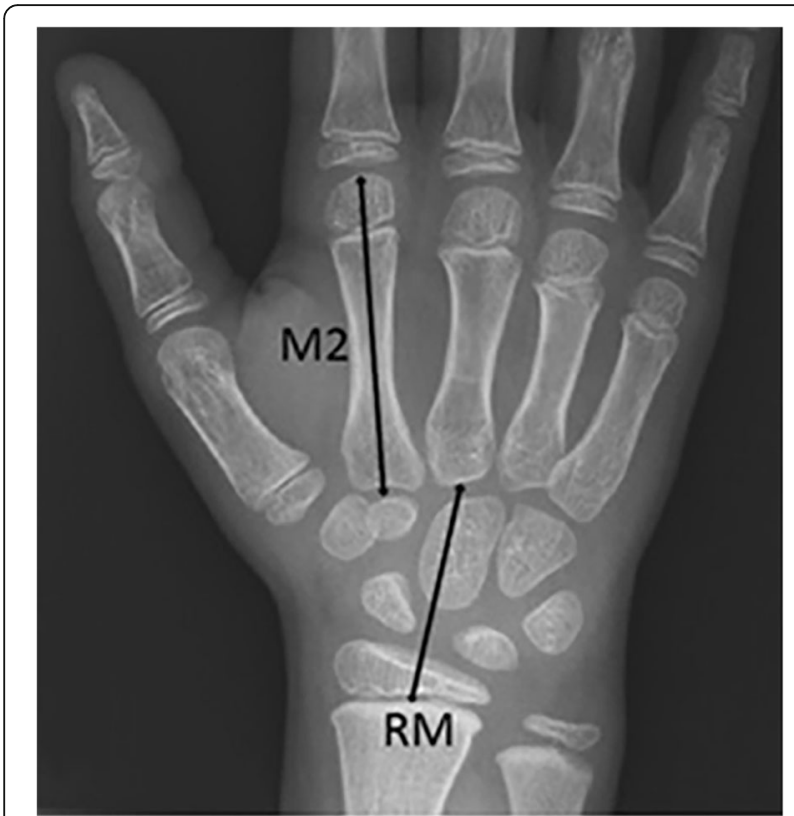

Fig. 2 Poznanski measurements used to determine the RM/M2 score. $\mathrm{RM}=$ radiometacarpal length; $\mathrm{M} 2$ = length of the second metacarpal

\section{Results}

\section{Patients}

Baseline characteristics of the included patients are presented in Table 1. Patients in arm 3 were younger and had longer symptom duration than patients in arm 1 and 2. Nine patients with radiographs initially did not have wrist arthritis, six patients never had wrist arthritis clinically.

Of the original 94 patients included in the BeSt-forKids cohort, 75 patients had at least 1 hand radiograph available. Overall, 268 radiographs were available. Sixteen radiographs, made outside the selected time frame, were left out, leaving 252 radiographs $(n=127$ of the left hand and $n=125$ of the right hand). Of these 92 (in 47 patients) were taken at baseline (with a window of 4 months before and 3 month after inclusion) and 160 (in 52 patients, 27 patients had more than 2 radiographs) during follow-up. Fourteen patients had closed growth plates at baseline and were left out, one patient left the study due to changing diagnosis and was not included in the current analysis [18]. A flow chart of the patient selection process is provided (Additional file 1).

Sixty patients with 252 radiographs (85 in arm 1, 79 in arm 2 and 88 in arm 3) were eligible for scoring by the Poznanski-method and BMD. For analysis of the BA 196 radiographs of 49 patients (65 in arm 1, 67 In arm 2, 64 in arm 3) were eligible, while $56 \mathrm{X}$-rays (20 in arm 1, 12 in arm 2 and 24 in arm 3) of 11 patients could not be scored because patients' age was $<5$ years.

\section{The Poznanski-score}

For the Poznanski-score the inter-observer correlations were 0.996 for RM and 0.999 for M2. The intra-observer correlations were $\geq 0.996$ for all measurements. Additional file 2 provides the Bland-Altman-plots.

At baseline, Poznanski-scores were comparable to those in healthy children, with a median Poznanski-score of $-0.45(-0.74-0.45)$. Over time, overall no significant change in Poznanski-score, unadjusted nor after adjusting for age and symptom duration, was observed, and there were no differences between the 3 arms (see Figs. 3 and 4 for observed and predicted changes in Poznanskiscore, BA and BMD Z-scores per arm). The outlier in Fig. 3b with a high Poznanski-score (Z-score 3,9) is a competing mountain-biker.

\section{Bone age}

At baseline, the mean BA Z-score was $0.04(-0.58 ; 0.67)$ for the entire group, similar to the normal reference population. Baseline scores in arm 3 were significantly lower than in arms 1 and 2, but still within the normal range (1 SD from 0 ). Over time there was a decrease in $\mathrm{BA}$ in arm 3 (arm 3 versus arm $1 p=0.024, \beta=0.014$ (95\%CI $-0.002 ; 0.027)$ which remained within the normal range (Fig. 4).

\section{Bone mineral density}

At baseline, the mean BMD Z-score was - 0.65 (-0.90; -0.40 ) for the entire group, with statistically significantly lower baseline BMD in arm 3 compared to the normal reference population. Over time the BMD, adjusted or unadjusted for symptom duration, remained unchanged in arm 1, showed a trend for increase in arm 2 and significantly increased in arm $3(p<0.001$ for arm 3 versus arm $1, \beta=-0.028$ (95\% CI $-0.043 ;-0.013)$.

Tables with detailed results of the LMM of the Poznanski-score, BA and BMD are presented in Additional file 3. Results comparing all left with all right hands, both at baseline and during follow-up, were not statistically different for Poznanski-score $(p=$ 0.809 , BA $(p=0.825)$ nor BMD $(p=0.404)$. Six patients with radiographs never had clinical inflammation of wrists. Sensitivity analyses excluding these patients showed similar results to the main analysis (Additional file 4A). Since we included $n=7$ patients with oligoarthritis and $n=3$ patients with psoriatic arthritis, numbers are too small to analyse these groups separately. Sensitivity analysis of the polyarticular subgroup only, showed similar results (Additional file 4B).

\section{Effect meanJADAS10-score over time on Poznanski, Bone Age and $B M D$}

To investigate whether mean JADAS10-score over time correlated with any of the radiological outcomes, we 
Table 1 Baseline characteristics of the patients selected from the original 3 arms

\begin{tabular}{|c|c|c|c|c|}
\hline & $\begin{array}{l}\text { Arm } 1 \\
\text { Sequential } \\
\text { monotherapy } \\
(n=21 / 31)\end{array}$ & $\begin{array}{l}\text { Arm } 2 \\
\text { Combo MTX } \\
+6 \text { wks Prednisone } \\
(n=18 / 32)\end{array}$ & $\begin{array}{l}\text { Arm } 3 \\
\text { Combo MTX } \\
+ \text { etanercept } \\
(n=21 / 29)\end{array}$ & $P$ \\
\hline Age (years), median (IQR) & $8.2(4.1 ; 10.2)$ & $7.9(5.7 ; 11.7)$ & $6.2(3.8 ; 10.4)$ & $<0.001$ \\
\hline Symptom duration (months), median (IQR) & $7.8(4.2 ; 11.3)$ & $5.3(2.6 ; 6.1)$ & $8.5(4.2 ; 12.1)$ & 0.015 \\
\hline ANA pos, n (\%) & $8(38)$ & $6(33)$ & $8(28)$ & 0.94 \\
\hline Female, n (\%) & $14(66.7)$ & $9(50)$ & $15(71.4)$ & 0.36 \\
\hline JIA Category: & & & & 0.90 \\
\hline Oligo, n (\%) & $3(14.3)$ & $2(11.1)$ & $2(9.5)$ & \\
\hline Poly, n (\%) & $17(81)$ & $14(77.8)$ & $18(85.7)$ & \\
\hline Psoriatic, n (\%) & $1(4.8)$ & $1(5.6)$ & $1(4.8)$ & \\
\hline VAS physician, mean \pm SD $(\mathrm{mm})$ & $43.6 \pm 15.7$ & $54.0 \pm 17.0$ & $52.9 \pm 17.5$ & 0.44 \\
\hline VAS patient/parent, mean \pm SD (mm) & $53 \pm 17.1$ & $56.8 \pm 23.4$ & $55.2 \pm 24.9$ & 0.31 \\
\hline $\mathrm{CHAQ}$, mean $\pm \mathrm{SD}$ & $0.95 \pm 0.7$ & $1.1 \pm 0.6$ & $1.0 \pm 0.6$ & 0.88 \\
\hline No. active joints, median (IQR) & $6(4.5 ; 14.5)$ & $8(5.8 ; 11.5)$ & $8(5.5 ; 11.5)$ & 0.56 \\
\hline No. limited joints, median (IQR) & $2(0.5 ; 4)$ & $1.5(0.8 ; 3.3)$ & $3(2.5 ; 5.5)$ & 0.68 \\
\hline ESR, median (IQR) (mm) & $6(2 ; 12)$ & $6(3.5 ; 32)$ & $9(6 ; 31.5)$ & 0.28 \\
\hline JADAS-10 mean \pm SD $(0-40)$ & $16.7 \pm 4.5$ & $19.6 \pm 5.1$ & $19.1 \pm 5.8$ & 0.24 \\
\hline Z-score Poznanski median (IQR) ${ }^{1}$ & $-0.45(-0.70 ; 0.56)$ & $-0.19(-0.57 ; 0.68)$ & $-0.61(-0.82 ; 0.17)$ & 0.056 \\
\hline Z-score Bone Age mean $(\mathrm{Cl})^{2}$ & $-0.38(-0.88 ; 0.11)$ & $0.51(0.28 ; 0.74)$ & $-0.43(-0.82 ;-0.04)$ & 0.001 \\
\hline Z-score BMD mean $(\mathrm{Cl})^{3}$ & $-0.44(-0.84 ;-0.04)$ & $-0.39(-0.93 ; 0.15)$ & $-1.08(-1.48 ;-0.68)$ & 0.03 \\
\hline Wrist arthritis, inclusion (\%) & 19/21 (90) & 14/18 (78) & 18/21 (86) & \\
\hline Wrist arthritis, follow-up (\%) & $17 / 21(81)$ & 14/18 (78) & $18 / 21(86)$ & \\
\hline Wrist arthritis, inclusion or follow-up (\%) & $21 / 21(100)$ & 14/18 (78) & 19/21 (90) & \\
\hline
\end{tabular}

JIA juvenile idiopathic arthritis, oligo oligoarticular JIA, poly polyarticular JIA, IQR interquartile range, VAS Visual Analogue Scale, ANA antinuclear antibody, RF rheumatoid factor, SD standard deviation, Cl confidence interval, CHAQ Child Health Assessment Questionnaire, No number, ESR Erythrocyte Sedimentation Rate, JADAS Juvenile Arthritis Disease Activity Score, BMD Bone Mineral Density, BA Bone Age (both using BoneXpert method) Z-scores were based on all available radiographs, including left and right hand radiographs. $1: n=35$ in arm $1, n=25$ for arm $2, n=31$ for arm $3 ; 2: n=16$ for arm $1, \mathrm{n}=18$ for arm 2 and $n=18$ for arm 3, 3: $n=33, n=25$ for arm 2 and $n=32$ for arm 3. $n=$ amount of $X$-rays

have performed separate analyses for the 3 radiological outcome measures. Mean JADAS10-score over time did not influence Poznanski score, $[\beta(95 \%$ CI $) 0.0010(-$ $0.00038 ; 0.0024), p=0.154]$, Bone Age $[\beta(95 \% \mathrm{CI})-0.00017$ $(-0.0018 ; 0.0014), p=0.84]$ or BMD [ $\beta$ (95\% CI) 0.00069 $(-0.0012 ; 0.0026), p=0.48]$. Tables and graphs from this analysis are reported in Additional file 4C.

\section{Discussion}

Our study is the first to describe longitudinal radiological outcomes of a tightly controlled treat-to-target approach, aimed at inactive disease during 24 months of treatment, in recent onset poly- and oligoarticular JIA patients. Despite a symptom duration of mean (SD) 7.6 (4.9) months and a JADAS-10 of 18.7 (5.6), at baseline, we found no significant differences in Poznanski-score and BA (as measured by the BoneXpert-method) of wrist radiographs compared to healthy children. Only in arm $3 \mathrm{BMD}$ as measured by the BoneXpert-method was significantly lower than the normal reference population.
After 24 months of treatment, there was no deterioration in any of the scores and in arm 3 BMD had statistically significantly improved. Mean JADAS10-scores over time were not associated with any of the radiological outcomes in this analysis.

Combined with rapid suppression of symptoms of active arthritis, prevention of damage is an important treatment goal in JIA. Damage has been most notably found in patients with longstanding and/or seropositive polyarticular JIA, but may also occur in seronegative polyarticular JIA and oligoarticular JIA [19-21]. As has been shown in rheumatoid arthritis, it is thought likely that, also in JIA, damage progression is driven by inflammatory processes. Assessing damage in patients who are in very different phases of joint development can be challenging. In growing children, cartilage thinning, delayed or accelerated growth and reduced bone mineral density rather than bony erosions and joint space narrowing may indicate damage. Decreased bone age often reflects delayed bony maturation in JIA [22, 23] but also 


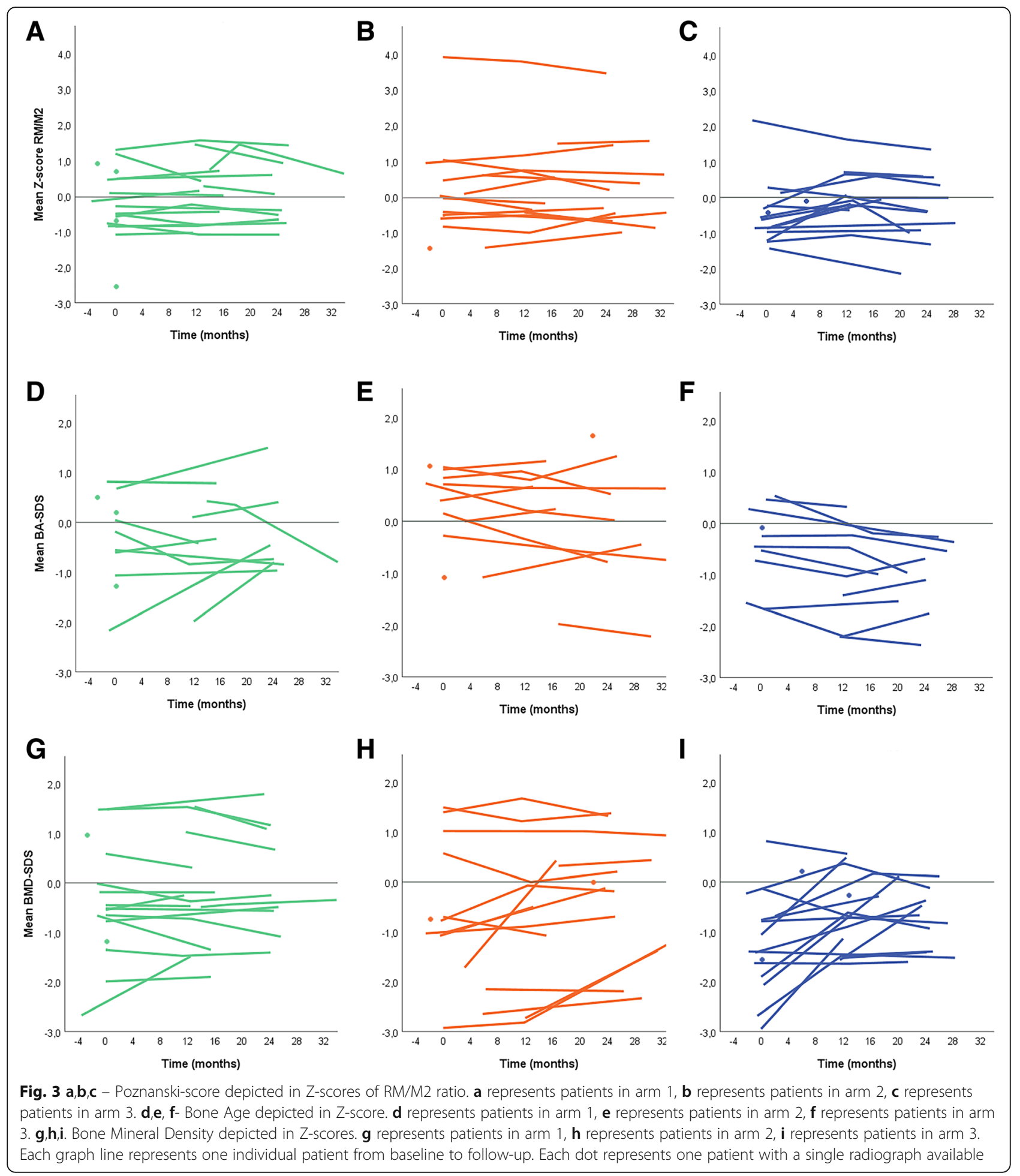

increased focal bone maturation can be a result of joint inflammation [15].

Compared to older cohorts [12, 24] or recent cohorts with longer disease duration [17], we found little damage at baseline in this cohort with recent-onset disease. Since we did not include patients with RF-positive polyarticular JIA, this could be a mildly affected cohort although initial JADAS10 scores were similar to other cohorts [25]. In addition we found no significant damage progression. This is possibly due to our strategy of tightly controlled treatment-to-target aiming at inactive disease in all 3 treatment arms, resulting in rapid suppression of inflammation in 


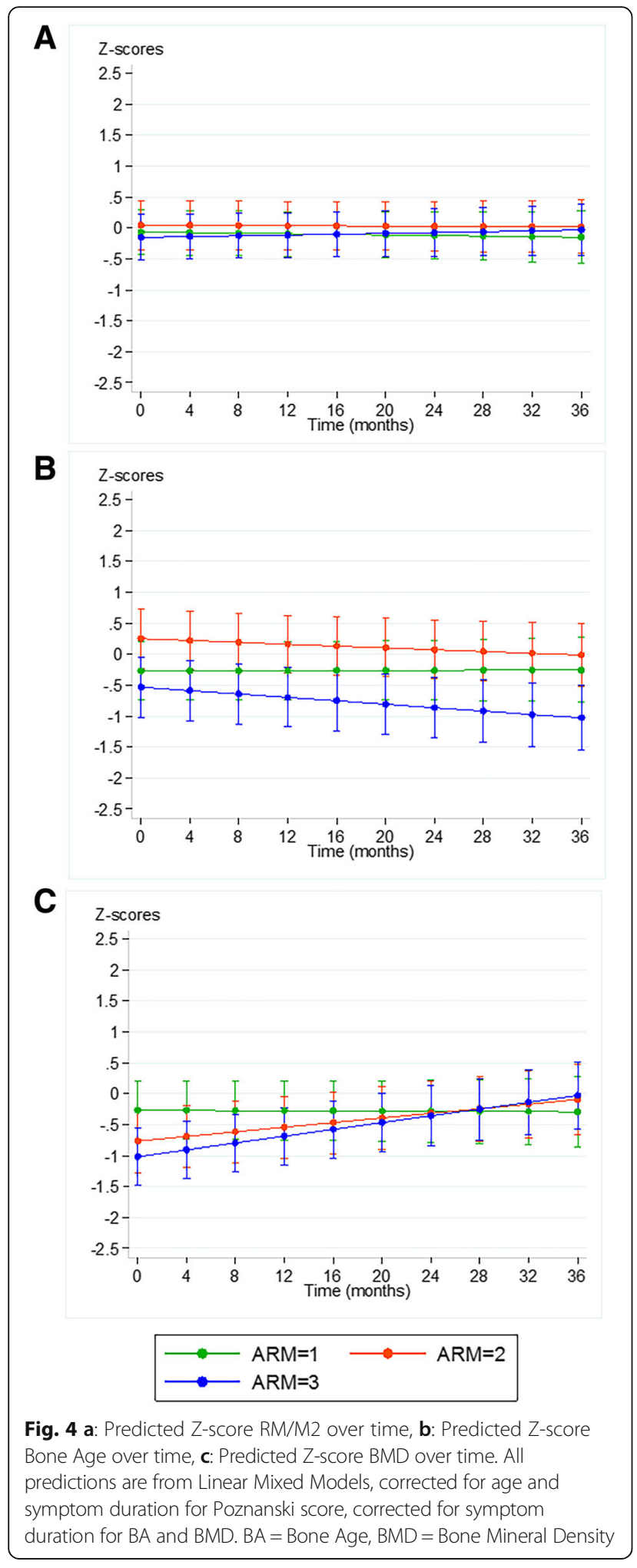

most patients, without significant differences between the strategy-arms after 24 months. Only in arm 3 there was an initial greater clinical improvement [10]. We cannot rule out an additional positive effect of use of etanercept, in all patients in arm 3, and in many in arms 1 and 2 after they failed to achieve remission on initial treatment with methotrexate (with or without temporary prednisone). Previous studies suggest that treatment with methotrexate cannot prevent joint damage progression whereas use of biologic DMARDs (used as initial treatment in our arm 3) may be more successful, although data are limited [26, 27]. Apart from strategy, we did not find an effect of mean JADAS10score over time, possibly in all patients due to rapid suppression of inflammation, therefore inhibiting the disease to have time to create damage.

To score differences in potentially little damage, we needed a sensitive scoring method. Conventional radiography has proven to be a useful modality to monitor wrist damage of JIA patients [2, 4, 5, 13, 28-31]. Several methods, like the Dijkstra-score [31], modified Sharp van der Heijde-score $[5,19]$ the modified Larsen-score $[4,32]$ and the Steinbrocker-scale [33, 34] have been developed to evaluate radiographic damage to the osteochondral structures of the wrist and hand. The Dijkstra composite-score is limited in the grading of changes for severity over time [35]. We stopped using the modified Sharp van der Heijde for pediatric assessment of joint damage [5] as it proved too difficult to uniformly score subtle changes in joint space narrowing, bony erosions and bone deformity, as was recognized previously [35]. Magnetic resonance imaging (MRI) and ultrasound (US) are suitable for monitoring disease activity for evaluating treatment response, and may also detect damage [36]. However, interpretation of MRI findings of the osteochondral domain in JIA patients is challenging due to characteristics of the growing skeleton, in particular in hand and wrist joints. Bone marrow edema and bony depressions are also frequently seen on MRI in wrists of healthy children [37-40]. Until now, no optimal method has been found to differentiate pathological and standardized age-specific findings in healthy children on MRI and US which limits their use to accurately assess damage and damage progression in the wrist of JIA patients.

The Poznanski-score, which measures relative carpal length on radiographs of the wrist, is able to detect deviating growth in absence of distinct joint space narrowing or erosions [13]. A disadvantage of the Poznanski-score is that it requires open growth plates, which caused ineligibility in 14 of our patients, and unreliability in case of carpometacarpal erosions which hampers discriminating bony ends, which did not occur in our cohort. In addition, we used the relatively new BoneXpert method to score Bone Age and BMD, which, compared to a healthy reference population, can indicate damage due to inflammation.

The BoneXpert method, based on digital X-ray radiogrammetry (DXR), allows to determine the Bone Age 
and BMD compared to a normal reference population, at lower costs and with lower radiation than manually comparing the hand radiograph with images in the atlas by Greulich and Pyle [16] and than measuring BMD by Dual Energy X-ray Absorptiometry (DXA) [41]. The BMD measurement by BoneXpert is corrected for the size of the cortical bones to compensate for the high variation in stature of growing children, in contrast to DXA. Previous studies have reported on delayed bone maturation as reflected by negative Z-scores for Bone Age [12, 17]. These studies had included patients with more severe or longstanding active disease. However, Borzutzky and others have warned previously, that determining bone age can be challenging in JIA due to accelerated maturation $[15,42]$.

In JIA patients, BMD is often reduced [12, 17, 43-45]. BMD was significantly lower at baseline in arm $3(-1.1$ SD, $(-1.48 ;-0.68)$ ). This could indicate longstanding or more severe disease. Indeed symptom duration in arm 3 was slightly longer than in arms 1 and 2, although JADAS10 scores at baseline were similar in the 3 arms. Possibly as a result of rapid and sustained suppression of inflammation, BMD improved significantly over time in arm 3. A previous study also reported improvement of BMD after therapy [45]. It is speculated that this improvement is due to the anti-inflammatory effect of DMARD treatment [46], more specifically due to etanercept $[47,48]$. However, no comparison cohort is available to prove that the treatment-to-target approach is responsible for a better radiological outcome.

Future studies are needed to delineate the effect of the treatment-to-target concept on improving bone health as reflected by bone maturation and BMD in JIA.

Our study has some limitations. Although comparable with other studies $[2,26,49]$ in children with JIA, we had a relatively small sample size $(n=60)$, and we may have lacked power to detect small differences. In previous studies, results were based on clinically inflamed wrists only. Since we have examined also 6 patients with wrist radiographs of unaffected wrists, in this study we may have underestimated damage, although sensitivity analyses excluding patients who never had any clinical wrist arthritis over 24 months showed similar results. It remains to be determined whether joint damage is mainly due to local inflammation or (also) to systemic inflammatory processes of JIA.

Due to our choice of scoring methods, patients were excluded who had closed growth plates. Also we disregarded results of radiographs made outside the selected time frame. Follow-up time was relatively short compared to previous cohorts. However, often radiographic damage is expected to occur within the first one or two years [2]. Finally, determination of bone health by BoneXpert software needs further validation, including further comparison with existing methods for the determination of BMD in JIA patients $[41,50,51]$.

\section{Conclusions}

We conclude that in our cohort of patients with recentonset JIA who were treated-to-target aiming at inactive disease, wrist-radiographs showed neither damage according to Poznanski at baseline, nor progression after 2 years. Bone age was within normal values at baseline and after follow-up. In arm 3, BMD was lower at baseline but improved significantly towards normalization during treatment. We propose that with earlier start of treatment and treatment to target, the focus of current treatment regimens shifts to damage prevention rather than suppression of damage progression. This will likely also prevent long-term disability. Future JIA-cohorts with more patients and longer follow-up are warranted to confirm these promising results for children with JIA.

\section{Additional files}

Additional file 1: Flow chart of patient selection process for the Poznanski-score. (DOCX 23 kb)

Additional file 2: Bland-Altman plots with 95\% limits of agreement. (DOCX 245 kb)

Additional file 3: $L M M$ for Poznanski, $B A$ and $B M D$ adjusted for age and/or symptom duration. (DOCX $14 \mathrm{~kb}$ )

Additional file 4: A Sensitivity analysis of patients with wrist arthritis, without $n=6$ with never wrist arthritis. B Sensitivity analysis of patients with polyarticular JIA. C LMM for mean JADAS10 score in relation to Poznanski, BA and BMD. (DOCX 804 kb)

\section{Abbreviations}

BMD: Bone Mineral Density; Cl: Confidence Interval; CR: Conventional Radiography; DMARD: Disease Modifying Antirheumatic Drug(s); ICC: Intraclass correlation coefficient; JIA: Juvenile Idiopathic Arthritis; M2: Second metacarpal; MRI: Magnetic resonance imaging; MTX: Methotrexate; RF: Rheumatoid Factor; RM: Radiometacarpal length; RMexp: Expected radiometacarpal length; RMobs: Observed radiometacarpal length; SSZ: Sulphasalazine; US: Ultrasound

\section{Acknowledgements}

We would like to thank all participating patients and parents. Stefan Böhringer, SB, biostatistician has advised us on the analysis of the data. Paul de Bruin, $\mathrm{PdB}$, independent computer technician of the radiology department served in anonymizing and randomizing all the $\mathrm{X}$-rays.

\section{Authors' contributions}

All authors were involved in drafting the article or revising it critically for important intellectual content. All authors approved the final version to be published. PCEHM had full access to all data in the study and takes responsibility for the integrity of the data and the accuracy of the data analysis. Study conception and design: PCEHM, WGvB, DS, SAB, RH, DMCB, CFA, LWAvSS, JMvdB, MAJvR, RtC, MM. Acquisition of data: PCEHM, DMCB, DSM, YKK, JMvdB, TWK, MAJvR, LWAvSS, CFA, RtC. Analysis and interpretation of data: WGVB, DS, PCEHM, CMN, SAB, RH, DMCB, LWAvSS, CFA, RtC, MM. Critically revising the manuscript; WGVB, DS, CMN, SAB, RH, DMCB, DSM, YKK, TWK, MAJvR, LWAvSS, JMvdB, CFA, RtC, MM

\section{Funding}

The BeSt for Kids study is an investigator-initiated study which received financia support from Pfizer, who had no role in study design, data collection, data 
analysis, data interpretation, writing of an abstract, or decision to submit a manuscript for submission. No other funding was required for this study.

\section{Availability of data and materials}

The datasets used and/or analyzed during the current study are available from the corresponding author on reasonable request.

\section{Ethics approval and consent to participate}

Approval of the Medical Ethical Committee of the Leiden University Medical Center and local Ethical Committees was obtained prior to start at each study site. Written Informed consent was obtained from patients above 12 years of age and parents of all participating patients.

\section{Consent for publication}

All authors agreed with the final version to be submitted. This study has not been published elsewhere, or is not currently submitted elsewhere.

\section{Competing interests}

The authors declare that they have no competing interests.

\section{Author details}

'Department of Pediatric Rheumatology, Leiden University Medical Center, Leiden, the Netherlands. ${ }^{2}$ Department of Pediatrics/Pediatric Rheumatology, Sophia Children's Hospital Erasmus Medical Center, J6-S, LUMC, PO Box 9600 2300 RC Leiden, Rotterdam, the Netherlands. ${ }^{3}$ Department of Radiology and Nuclear Medicine, Amsterdam University Medical Center, Amsterdam Movement Sciences, Amsterdam University Medical Center, Amsterdam, the Netherlands. ${ }^{4}$ Department of Pediatric, Immunology, Rheumatology and Infectious Diseases, Emma Children's Hospital, Amsterdam University Medical Center, Amsterdam, the Netherlands. ${ }^{5}$ Department of Pediatrics, Hagaziekenhuis Juliana Children's Hospital, the Hague, the Netherlands. ${ }^{6}$ Department of Pediatric Rheumatology, Amsterdam Rheumatology and Immunology Center location Reade, Amsterdam, the Netherlands. ${ }^{7}$ Department of Pediatrics, Emma Children's Hospital AMC, Amsterdam University Medical Center, Amsterdam, the Netherlands. ${ }^{8}$ Department of Rheumatology, Leiden University Medical Center, Leiden, the Netherlands.

Received: 18 April 2019 Accepted: 9 August 2019

Published online: 04 September 2019

\section{References}

1. Petty RE, Southwood TR, Manners P, Baum J, Glass DN, Goldenberg J, et al. International league of associations for rheumatology classification of juvenile idiopathic arthritis: second revision, Edmonton, 2001. J Rheumatol. 2004;31(2):390-2.

2. Magni-Manzoni S, Rossi F, Pistorio A, Temporini F, Viola S, Beluffi G, et al Prognostic factors for radiographic progression, radiographic damage, and disability in juvenile idiopathic arthritis. Arthritis Rheum. 2003;48(12):3509-17.

3. Adib N, Silman A, Thomson W. Outcome following onset of juvenile idiopathic inflammatory arthritis: II. Predictors of outcome in juvenile arthritis. Rheumatology (Oxford). 2005;44(8):1002-7.

4. Rossi F, Di Dia F, Galipo O, Pistorio A, Valle M, Magni-Manzoni S, et al. Use of the sharp and Larsen scoring methods in the assessment of radiographic progression in juvenile idiopathic arthritis. Arthritis Rheum. 2006:55(5):717-23.

5. Ravelli A, loseliani M, Norambuena X, Sato J, Pistorio A, Rossi F, et al. Adapted versions of the sharp/van der Heijde score are reliable and valid for assessment of radiographic progression in juvenile idiopathic arthritis. Arthritis Rheum. 2007:56(9):3087-95.

6. Bertamino M, Rossi F, Pistorio A, Lucigrai G, Valle M, Viola S, et al. Development and initial validation of a radiographic scoring system for the hip in juvenile idiopathic arthritis. J Rheumatol. 2010;37(2):432-9.

7. Ringold S, Seidel KD, Koepsell TD, Wallace CA. Inactive disease in polyarticular juvenile idiopathic arthritis: current patterns and associations. Rheumatology (Oxford). 2009;48(8):972-7.

8. Ravelli A, Consolaro A, Horneff G, Laxer RM, Lovell DJ, Wulffraat NM, et al. Treating juvenile idiopathic arthritis to target: recommendations of an international task force. Ann Rheum Dis. 2018;77(6):819-28.

9. Beukelman T, Patkar NM, Saag KG, Tolleson-Rinehart S, Cron RQ, DeWitt EM, et al. 2011 American College of Rheumatology recommendations for the treatment of juvenile idiopathic arthritis: initiation and safety monitoring of therapeutic agents for the treatment of arthritis and systemic features. Arthritis Care Res (Hoboken). 2011:63(4):465-82.

10. Hissink Muller PC, Brinkman DM, Schonenberg D, Koopman-Keemink Y, Brederije IC, Bekkering WP, et al. A comparison of three treatment strategies in recent onset non-systemic juvenile idiopathic arthritis: initial 3-months results of the BeSt for kids-study. Pediatr Rheumatol Online J. 2017:15(1):11.

11. Hissink Muller $P$, Brinkman DMC, Schonenberg-Meinema $D$, van den Bosch WB, Koopman-Keemink Y, Brederije ICJ, et al. Treat to target (drug-free) inactive disease in DMARD-naive juvenile idiopathic arthritis: 24-month clinical outcomes of a three-armed randomised trial. Ann Rheum Dis. 2019; 78(1):51-9.

12. Anink J, Nusman CM, van Suijlekom-Smit LW, van Rijn RR, Maas M, van Rossum MA. Automated determination of bone age and bone mineral density in patients with juvenile idiopathic arthritis: a feasibility study. Arthritis Res Ther. 2014;16(4):424

13. Poznanski AK, Hernandez RJ, Guire KE, Bereza UL, Garn SM. Carpal length in children--a useful measurement in the diagnosis of rheumatoid arthritis and some concenital malformation syndromes. Radiology. 1978;129(3):661-8.

14. Garn SM, Hertzog KP, Poznanski AK, Nagy JM. Metacarpophalangeal length in the evaluation of skeletal malformation. Radiology. 1972;105(2):375-81.

15. Borzutzky A, Martinez-Aguayo A. Accelerated carpal bone maturation in juvenile idiopathic arthritis: pitfall for bone age measurement. J Pediatr Endocrinol Metab. 2011:24(7-8):551.

16. Greulich WP, SI. Radiographic atlas of the skeletal development of the hand and wrist. Stanford: Stanford University Press; 1959.

17. Twilt M, Pradsgaard D, Spannow AH, Horlyck A, Heuck C, Herlin T. Joint cartilage thickness and automated determination of bone age and bone health in juvenile idiopathic arthritis. Pediatr Rheumatol Online J. 2017;15(1):63.

18. Hissink Muller PCE, Donze SH, ten Cate R. Polyarteritis Nodosa Mimicking Juvenile Idiopathic Arthritis: A Case Report. Ann Paediatr Rheum. 2014:3(3): 141-5. https://doi.org/10.5455/apr.091520140648.

19. Giancane G, Pederzoli S, Norambuena X, loseliani M, Sato J, Gallo MC, et al. Frequency of radiographic damage and progression in individual joints in children with juvenile idiopathic arthritis. Arthritis Care Res (Hoboken). 2014;66(1):27-33

20. Cassone R, Falcone A, Rossi F, Magni-Manzoni S, Felici E, Buoncompagni A, et al. Unilateral destructive wrist synovitis in juvenile idiopathic arthritis. Clin Exp Rheumatol. 2004;22(5):637-42.

21. Anink J, Dolman KM, Merlijn van den Berg J, van Veenendaal M, Kuijpers TW, van Rossum MA. Two-year outcome of juvenile idiopathic arthritis in current daily practice: what can we tell our patients? Clin Exp Rheumatol. 2012;30(6): 972-8.

22. Stagi S, Masi L, Capannini S, Cimaz R, Tonini G, Matucci-Cerinic M, et al. Cross-sectional and longitudinal evaluation of bone mass in children and young adults with juvenile idiopathic arthritis: the role of bone mass determinants in a large cohort of patients. J Rheumatol. 2010;37(9):1935-43.

23. Burnham JM, Leonard MB. Bone disease in pediatric rheumatologic disorders. Curr Rheumatol Rep. 2004;6(1):70-8.

24. Thornton J, Pye SR, O'Neill TW, Rawlings D, Francis RM, Symmons DP, et al. Bone health in adult men and women with a history of juvenile idiopathic arthritis. J Rheumatol. 2011:38(8):1689-93.

25. Horneff G, Klein A, Klotsche J, Minden K, Huppertz HI, Weller-Heinemann F, et al. Comparison of treatment response, remission rate and drug adherence in polyarticular juvenile idiopathic arthritis patients treated with etanercept, adalimumab or tocilizumab. Arthritis Res Ther. 2016:18(1):272.

26. Nielsen S, Ruperto N, Gerloni V, Simonini G, Cortis E, Lepore L, et al. Preliminary evidence that etanercept may reduce radiographic progression in juvenile idiopathic arthritis. Clin Exp Rheumatol. 2008;26(4):688-92.

27. Avenarius DFM, Nusman C, Malattia C, de Horatio LT, Rosendahl K, Maas M, et al. Current status of wrist imaging in juvenile idiopathic arthritis. Pediatr Radiol. 2018;48(6):801-10.

28. Ravelli A, Viola S, Ramenghi B, Beluffi G, Zonta LA, Martini A. Radiologic progression in patients with juvenile chronic arthritis treated with methotrexate. J Pediatr. 1998:133(2):262-5.

29. Mason T, Reed AM, Nelson AM, Thomas KB, Patton A, Hoffman AD, et al. Frequency of abnormal hand and wrist radiographs at time of diagnosis of polyarticular juvenile rheumatoid arthritis. J Rheumatol. 2002;29(10):2214-8.

30. van Rossum MA, Zwinderman AH, Boers M, Dijkmans BA, van Soesbergen RM, Fiselier TJ, et al. Radiologic features in juvenile idiopathic arthritis: a first step in the development of a standardized assessment method. Arthritis Rheum. 2003;48(2):507-15. 
31. van Rossum MA, Boers $M$, Zwinderman $A H$, van Soesbergen $R M$, Wieringa $H$, Fiselier TJ, et al. Development of a standardized method of assessment of radiographs and radiographic change in juvenile idiopathic arthritis: introduction of the Dijkstra composite score. Arthritis Rheum. 2005;52(9):2865-72.

32. Aoki C, Inaba Y, Choe H, Kaneko U, Hara R, Miyamae T, et al. Discrepancy between clinical and radiological responses to tocilizumab treatment in patients with systemic-onset juvenile idiopathic arthritis. J Rheumatol. 2014;41(6):1171-7.

33. Michels H, Hafner R, Morhart R, Schuchmann L, Truckenbrodt H. Five year follow-up of a prospective cohort of juvenile chronic arthritis with recent onset. Clin Rheumatol. 1987;6(Suppl 2):87-92.

34. Lipinska J, Brozik H, Stanczyk J, Smolewska E. Anticitrullinated protein antibodies and radiological progression in juvenile idiopathic arthritis. J Rheumatol. 2012;39(5):1078-87.

35. Ravelli A. The time has come to include assessment of radiographic progression in juvenile idiopathic arthritis clinical trials. J Rheumatol. 2008;35(4):553-7.

36. Ventura-Rios L, Faugier E, Barzola L, De la Cruz-Becerra LB, Sanchez-Bringas G, Garcia AR, et al. Reliability of ultrasonography to detect inflammatory lesions and structural damage in juvenile idiopathic arthritis. Pediatr Rheumatol Online J. 2018;16(1):58.

37. Muller LS, Avenarius D, Damasio B, Eldevik OP, Malattia C, Lambot-Juhan K, et al. The paediatric wrist revisited: redefining MR findings in healthy children. Ann Rheum Dis. 2011;70(4):605-10.

38. Avenarius DM, Ording Muller LS, Eldevik P, Owens CM, Rosendahl K. The paediatric wrist revisited--findings of bony depressions in healthy children on radiographs compared to MRI. Pediatr Radiol. 2012;42(7):791-8.

39. Avenarius DF, Ording Muller LS, Rosendahl K. Erosion or normal variant? 4year MRI follow-up of the wrists in healthy children. Pediatr Radiol. 2016; 46(3):322-30.

40. Ording, Muller LS, Boavida P, Avenarius D, Damasio B, Eldevik OP, Malattia C, et al. MRI of the wrist in juvenile idiopathic arthritis: erosions or normal variants? A prospective case-control study. Pediatr Radiol. 2013;43:785-95.

41. van Rijn RR, Van Kuijk C. Of small bones and big mistakes; bone densitometry in children revisited. Eur J Radiol. 2009;71(3):432-9.

42. Skorpik G, Koob E, Grill F. Premature maturation of the carpal bones as an early diagnostic sign of juvenile rheumatoid arthritis. Handchir Mikrochir Plast Chir. 1991;23(4):202-6.

43. Lien G, Flato B, Haugen M, Vinje O, Sorskaar D, Dale K, et al. Frequency of osteopenia in adolescents with early-onset juvenile idiopathic arthritis: a long-term outcome study of one hundred five patients. Arthritis Rheum. 2003;48(8):2214-23.

44. Tang $T$, Tang $X, X u L$, Huang $Y$, Zeng J, Li Q. Evaluation of bone mass in children and young adults with juvenile idiopathic arthritis. Clin Exp Rheumatol. 2015;33(5):758-64.

45. Stagi S, Cavalli L, Signorini C, Bertini F, Cerinic MM, Brandi ML, et al. Bone mass and quality in patients with juvenile idiopathic arthritis: longitudinal evaluation of bone-mass determinants by using dual-energy $x$-ray absorptiometry, peripheral quantitative computed tomography, and quantitative ultrasonography. Arthritis Res Ther. 2014;16(2):R83.

46. Reed AM, Haugen M, Pachman LM, Langman CB. Repair of osteopenia in children with juvenile rheumatoid arthritis. J Pediatr. 1993;122(5 Pt 1):693-6.

47. Simonini G, Giani T, Stagi S, de Martino M, Falcini F. Bone status over 1 yr of etanercept treatment in juvenile idiopathic arthritis. Rheumatology (Oxford). 2005;44(6):777-80

48. Billiau AD, Loop M, Le PQ, Berthet F, Philippet P, Kasran A, et al. Etanercept improves linear growth and bone mass acquisition in MTX-resistant polyarticular-course juvenile idiopathic arthritis. Rheumatology (Oxford). 2010:49(8):1550-8.

49. Harel L, Wagner-Weiner L, Poznanski AK, Spencer CH, Ekwo E, Magilavy DB. Effects of methotrexate on radiologic progression in juvenile rheumatoid arthritis. Arthritis Rheum. 1993;36(10):1370-4.

50. Picaud JC, Duboeuf F, Vey-Marty V, Delams P, Claris O, Salle BL, et al. First all-solid pediatric phantom for dual X-ray absorptiometry measurements in infants. J Clin Densitom. 2003;6(1):17-23.

51. Nusman CM, Anink J, Otten MH, van Rossum MA, van Rijn RR, Maas M, et al. Bone health of patients with juvenile idiopathic arthritis: a comparison between dual-energy $\mathrm{X}$-ray absorptiometry and digital $\mathrm{X}$-ray radiogrammetry. Eur J Radiol. 2015;84(10):1999-2003.

\section{Publisher's Note}

Springer Nature remains neutral with regard to jurisdictional claims in published maps and institutional affiliations.

\section{Ready to submit your research? Choose BMC and benefit from}

- fast, convenient online submission

- thorough peer review by experienced researchers in your field

- rapid publication on acceptance

- support for research data, including large and complex data types

- gold Open Access which fosters wider collaboration and increased citations

- maximum visibility for your research: over $100 \mathrm{M}$ website views per year

At BMC, research is always in progress.

Learn more biomedcentral.com/submissions 